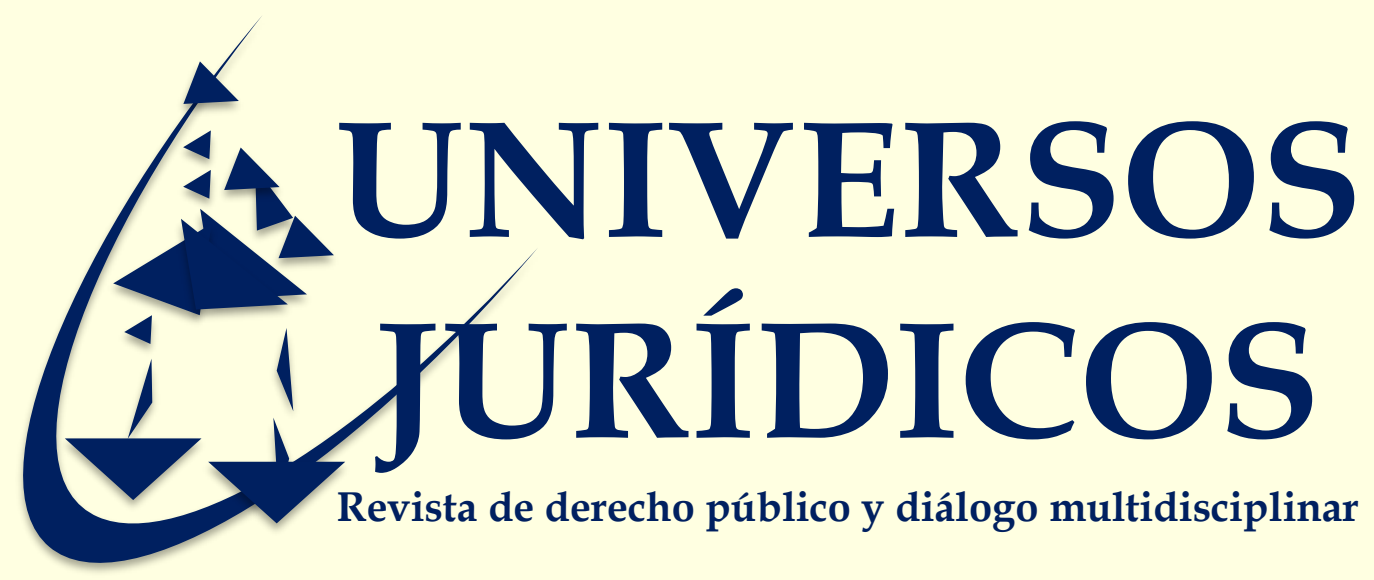

\title{
LA IMPORTANCIA DE LA TEORÍA DEL CASO EN EL NUEVO SISTEMA DE JUSTICIA PENAL.
}

\author{
Dr. Alejandro de la Fuente Alonso*
}

* Maestro en Economía, Doctor en Derecho por la UNAM, Miembro del Sistema Nacional de Investigadores, Investigador de la Universidad Veracruzana.

UNIVERSOS JURÍDICOS. Revista de derecho público y diálogo multidisciplinar. Año 6, No. 10, mayo 2018-octubre 2018, ISSN 2007-9125

Cómo citar este artículo en formato APA De la Fuente, A. (2018). La importancia de la teoría del caso en el nuevo sistema de justicia penal. Universos Jurídicos, 1-24.

Fecha de recepción: 27 de febrero de 2018. Fecha de aceptación: 05 agosto de 2018. 


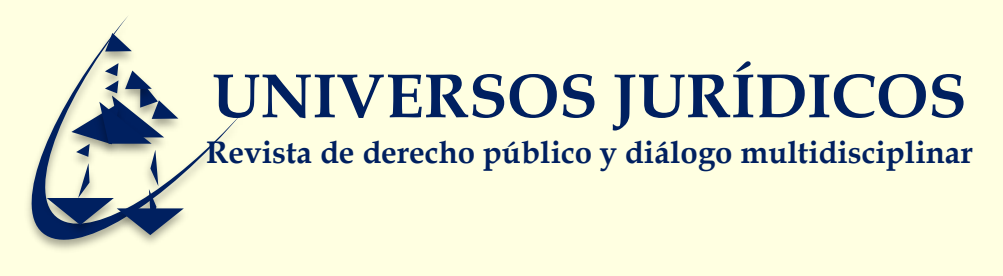

SUMARIO: I. INTRODUCCIÓN, II. ¿QUÉ ES LA TEORÍA DEL CASO?, III. ELEMENTOS DE LA TEORIA DEL CASO, IV. FASES, V. UTILIDAD DE LA TEORIA DEL CASO, VI. SUJETOS PROCESALES, VII. PONDERACIÓN, VIII. CONCLUSIÓN, IX. REFERENCIAS.

\section{RESUMEN}

En el presente trabajo se aborda en forma monográfica y analítica el concepto de la teoría del caso, utilizando como fuente diversos artículos de la doctrina jurídica, con el objetivo de esclarecer su concepto e importancia en virtud de la vigencia y puesta en marcha del nuevo Sistema de Justicia Penal Acusatorio, para ello se estudia su concepto, elementos, fases, utilidad y relevancia para los sujetos procesales.

\section{PALABRAS CLAVE}

Teoría del caso, Sistema de justicia penal, Proceso penal. 


\section{B. UNIVREOS IURRIIICOS}

\section{ABSTRACT}

In the present work, the concept of the theory of the case is approached in a monographic and analytical way, using various articles of legal doctrine as a source, with the aim of clarifying its concept and importance by virtue of the validity and implementation of the new System. of Accusatory Criminal Justice, for this purpose its concept, elements, phases, utility and relevance for procedural subjects are studied.

\section{KEYWORDS}

Case theory, Criminal justice system, Criminal process.

\section{INTRODUCCIÓN}

Con las reformas constitucionales a los artículos 16, 17, 18, 19, 20 y 21 publicadas el 18 de junio del 2008 en el Diario Oficial de la Federación, se da entrada a un nuevo sistema procesal de justicia que busca implementar distintos mecanismos de fortalecimiento del debido proceso, teniendo como fin una manera más eficaz y 


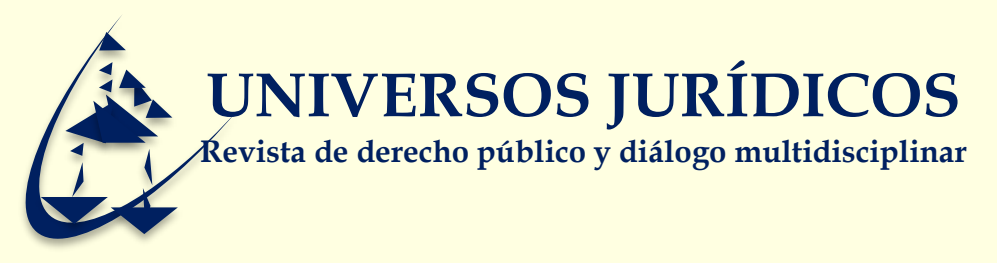

justa de impartir la justicia. Lo que implica que se deja en el pasado los procesos escritos, lentos y que retardaban los resultados.

Se da un cambio de paradigma en la forma de impartir justicia, buscando con esto una manera distinta de llevar a cabo los procesos, en donde ahora se tiene como principal objetivo la presencia del juzgador para que con ello se dé un resultado imparcial.

El objetivo principal de este trabajo es presentar un esbozo de la importancia de la teoría del caso en nuestro sistema penal, al ser esta de mayor relevancia para el desarrollo del juicio y de sus diferentes etapas.

Abordar las diferentes posturas de los sujetos procesales que intervienen en el desenlace es una tarea que no resulta sencilla, debido a que además de los cambios legales, estructurales y materiales que implica tal transformación, la principal exigencia del sistema, genera un gran reto ya que cada actor en el transcurso del juicio debe asumir de manera responsable el papel que le corresponde; a pesar de ello, en el proceso hacía la implementación se generan diversas preguntas, tales son: ¿podrá el ministerio publico asumir con 


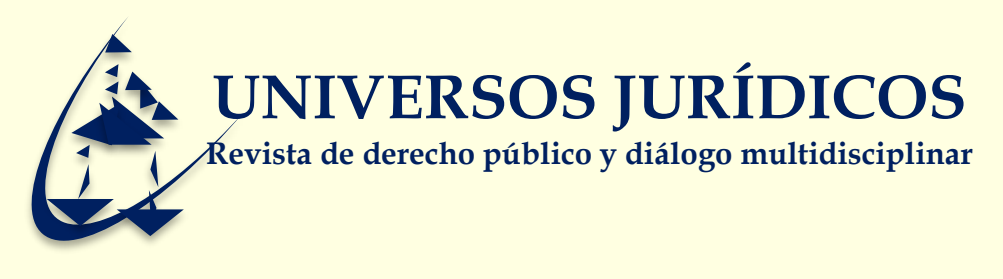

responsabilidad su tarea en el sistema adversarial que es el de investigar y acusar?, ¿los abogados litigantes podrán ejercitar una defensa adecuada? Y la más importante, ¿el juez decidirá la culpabilidad únicamente con los elementos que aportaron las partes, sin interesarse sobre la verdad histórica?

La teoría del caso surge como un instrumento de tal transcendencia para el pleno desenvolvimiento y eficaz desarrollo del proceso penal, al mismo tiempo es de suma importancia ya que es una herramienta básica para que el caso tenga el impacto necesario, ya que debe existir un pensamiento lógico jurídico en el momento de argumentar oralmente en las diversas etapas para obtener de ellas el mayor beneficio, según sean las pretensiones procesales que se tenga $n$.

\section{II. ¿QUÉ ES LA TEORÍA DEL CASO?}

En el sistema de justicia se necesita principalmente de un hecho delictuoso para después ir conociendo de los sucesos más relevantes e importantes y con todo lo investigado las partes tengan cómo realizar su teoría del caso.

Según Olga Fernanda Casarez Zazueta y German Guillen López, la teoría del caso es un planteamiento metodológico que cada una de las partes debe de realizar 


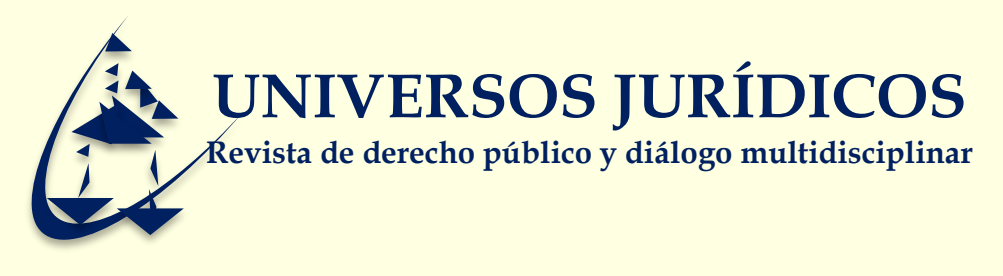

desde el primer momento que han tomado conocimiento de los hechos, con la finalidad de dotar de un solo sentido, significado u orientación de los hechos, normas jurídicas, sustantivas y procesales, así como el material probatorio, también conocido como evidencia. ( ://www.juridicaformativa.uson.mx)

Para el autor de la obra titulada "manual para litigantes del procedimiento nacional acusatorio y oral", de Eduardo Martínez - Bastida, la teoría del caso se utiliza para aludir a la metodología a usar por cada una de las partes dentro del proceso penal a los efectos de analizar ámbitos facticos, probatorios y jurídicos; puede decirse que la teoría del caso es el planteamiento de la parte que acusa o de la defensa respecto de: (2016)
a) hechos penalmente relevantes;
b) las pruebas que lo sustentan;
y c) los fundamentos jurídicos. 


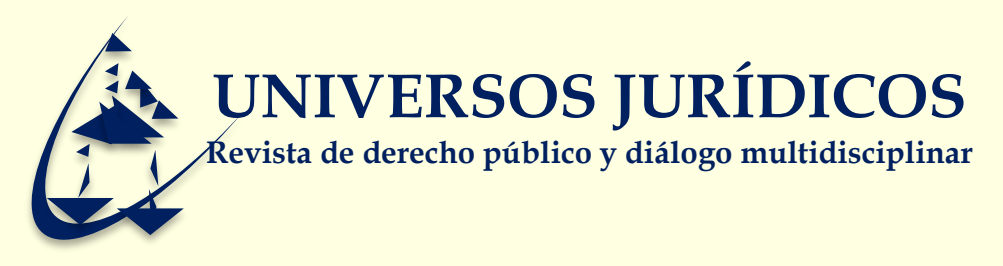

\section{ELEMENTOS DE LA TEORIA DEL CASO}

Para poder formular la teoría del caso se tiene que contar con tres elementos fundamentales, ya que con ellos se le dará un orden y dirección a tal teoría, y estos deben de beneficiar a las partes y a su vez al juez, por eso son indispensables para la realización de la teoría del caso. Los cuales son:

\section{a. ELEMENTO FACTICO}

Es la identificación de los hechos relevantes o conducentes que deben ser reconstruidos durante el debate oral, a través de las pruebas. Los hechos contienen la acción o acciones realizadas de tiempo, modo o lugar, los instrumentos utilizados, y el resultado de la acción o acciones realizadas.

Se puede contar con múltiples proposiciones fácticas para cada uno de los elementos legales, o solo con una. Estas proposiciones pueden ser fuertes o débiles. 


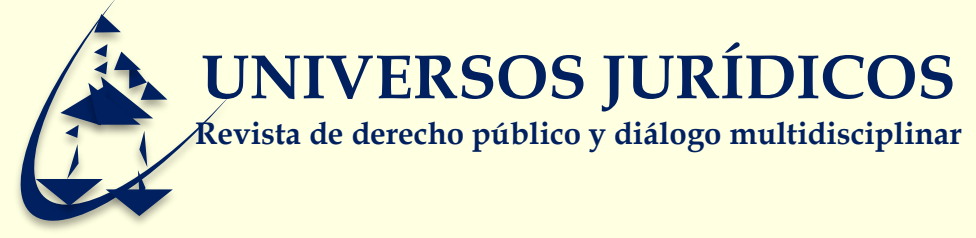

\section{b. ELEMENTO JURIDICO}

Consiste en dos fases. La primera tiene como objetivo, por un lado, determinar la ley penal aplicable; y, por otro lado, la teoría jurídica a ser empleada en el caso. La segunda fase consiste en examinar los elementos de la conducta punible; esto es la subsunción de los hechos en cada uno de los elementos de la teoría jurídica seleccionada. (Chorres, 2012)

\section{c. ELEMENTO PROBATORIO}

Sustenta lo factico; permite establecer cuáles son las pruebas convenientes que soporten la conducta punible y de responsabilidad del acusado, o la ausencia o falla de estos requisitos en el caso de la defensa.

\section{FASES}

Para el ordenamiento y estructura de la teoría se tiene la necesidad de desarrollar criterios para la metodología de una integración del caso eficiente, en la cual cada uno de las partes de forma particular darán su perspectiva de formación académica y de campo, y esto lo desarrollarán con una secuencia lógica de un procedimiento de investigación, siguiendo seis fases, las cuales son: 


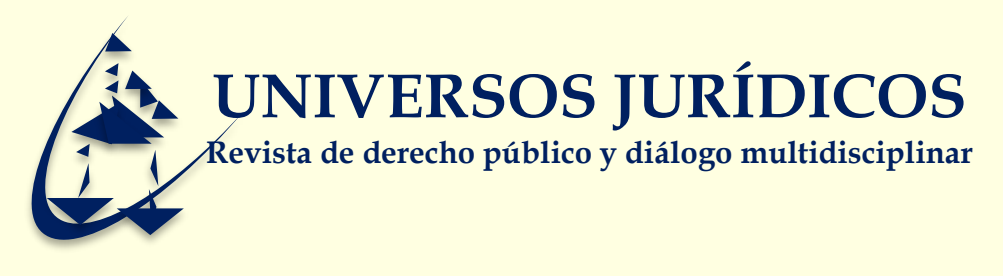

\section{a. PRIMERA FASE: CONOCIMIENTO GENERAL DEL CASO.}

Es necesario conocer tanto como sea posible sobre el caso inclusive aun aquellos elementos que pudieran parecer a simple vista irrelevantes o no relacionados siempre con una representación en nuestros sentidos de evitar pre-juiciarse, aun frente al peor de los sucesos, éticamente el abogado defensor o el ministerio publico están obligados a mantener un status neutral aunque parezca imposible, si nuestra percepción se daña por la apreciación o priori del hecho delictuoso frente a nuestros ojos, se alteran nuestros sentidos y por la tanto la dirección de la investigación. (Ortiz, 2014)

\section{b. SEGUNDA FASE: GENERACIÓN DE ELEMENTOS BÁSICOS DE INFORMACIÓN.}

Realizado el recuentro general considerando todas las peculiaridades del caso, empezaremos con el proceso de discriminación, quiere decir que todo aquello que no sea plausible como parte de la investigación que ha de realizarse se desechó para optimizar tiempo, por ejemplo:

Sucesos pocos importantes o intrascendentes para la investigación.

UNIVERSIDAD VERACRUZANA.

Instituto de Investigaciones Jurídicas

http://universosjuridicos.uv.mx/index.php/univerjuridicos/index

Xalapa, Veracruz, México 


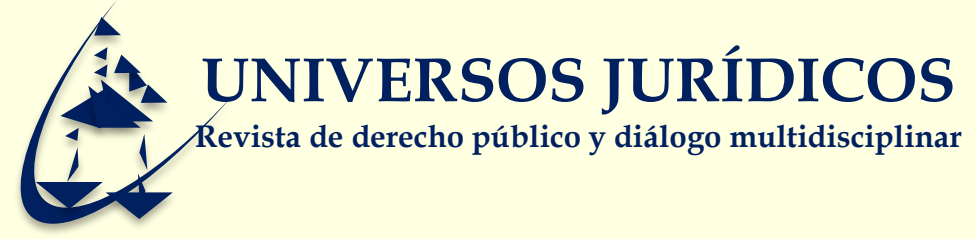

Pruebas encaminadas a desviar la atención del investigador.

Narraciones ociosas realizadas por personas que ni aportan datos contundentes.

\section{c. TERCERA FASE: BÚSQUEDA Y OBTENCIÓN DE ELEMENTOS BÁSICOS DE INFORMACIÓN.}

A partir del análisis general, previo de la información obtenida se establece un orden de prioridades de la investigación dirigido a:

Lugares a visitar para confirmar la versión de los hechos, es muy importante acudir al lugar de los hechos en la hora y en las condiciones en que se desarrolló el incidente, no quiere decir que con ello que si el registro señala el 24 de diciembre del año en curso, tendremos que esperar hasta el próximo año para acudir, sino que en la medida de la posible ubicarnos en el tiempo y lugar de ocurrencia, por ejemplo en un caso de un robo con violencia a casa habitación, la hora y el día son fundamentales, los testigos y las condiciones son casi constantes, debido a que personas que circulan en sus vehículos, o transeúntes o vecinos, realizan determinadas actividades en la misma fecha y hora. 


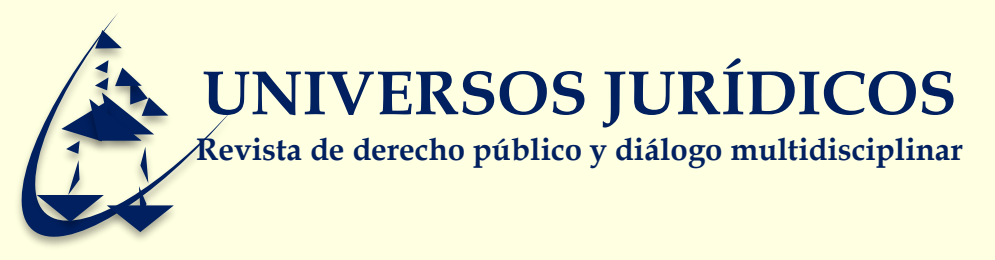

Personas a entrevistar si son compañeros de escuela, del trabajo o amistades con quien la víctima o el imputad o comparten determinadas actividades, tomando en cuenta los tiempos de intervención, pues mientras más lejano sea independientemente a que pueden ser objetados, su memoria se altera perdiendo datos claves para nuestra investigación. (Ortiz, 2014)

\section{d. CUARTA FASE: ANÁLISIS DE ELEMENTOS BÁSICOS DE INFORMACIÓN.}

Una vez obtenidos los datos necesarios dentro de nuestra investigación deben ser analizados en cuanto a su valor dentro del proceso:

- La credibilidad de un testigo y verosimilitud de sus manifestaciones. La autenticidad de un documento.

- La factibilidad de que quien produce un testimonio no se encuentra motivado por una causa externa o distinta a la simple aportación de datos.

- Si la evidencia ha cumplido con la cadena de custodia. 


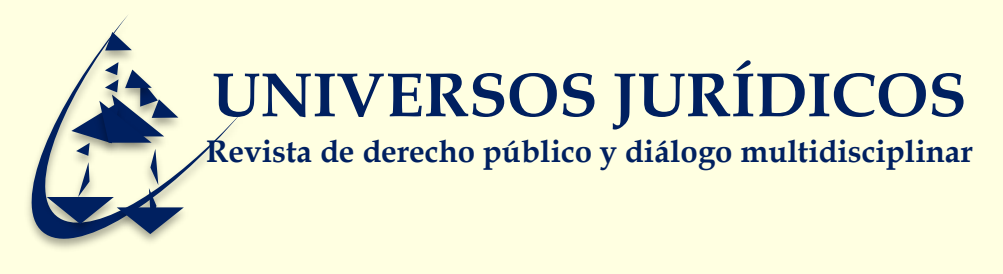

- Si la evidencia ha sido recolectada de acuerdo a lineamientos técnicos para su tratamiento y embalaje.

e. QUINTA FASE: CLASIFICACIÓN Y DEPURACIÓN DE LOS ELEMENTOS BÁSICOS DE LA INFORMACIÓN, DE EXPLOTACIÓN MEDIATA O INMEDIATA ESTABLECIENDO UN ORDEN DE PRIORIDADES LIGADO A LA NATURALEZA DE LOS ELEMENTOS PROBATORIOS.

Toda investigación lleva orden o cronología, se sugiere elaborar un cronograma de actuaciones que considere los términos procesales, así como los tiempos propios para su desarrollo, aun cuando la ley adjetiva nos señale periodos precisos que desde luego son determinados por el órgano jurisdiccional, no establece la forma y condiciones en que se desarrollaran las actividades de investigación.

f. SEXTA FASE: CONFIGURACIÓN DE LA TEORÍA DEL CASO CON LOS ELEMENTOS FACTICOS DEPURADOS. 


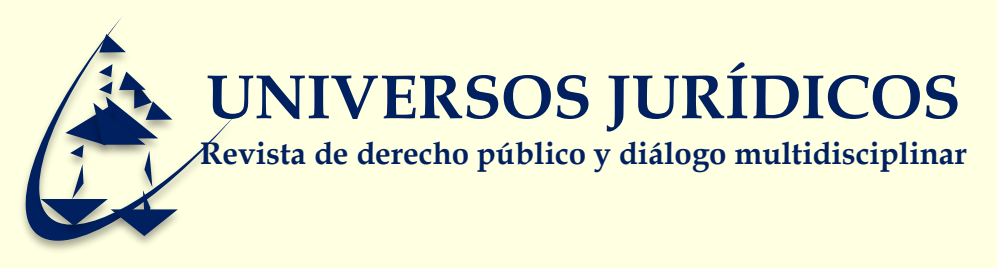

Una vez concluido el proceso de investigación fáctica, pasamos a la integración de la teoría del caso que analizados en su conjunto deberán cumplir con las características de viabilidad.

Para la construcción de esta teoría fáctica, se toman los hechos de una manera secuencial, a su vez se hacen de una forma coherente y de forma conveniente teniendo en cuenta el posible motivo o explicación de los hechos, para que con eso se reconstruya la historia de la teoría planteada.

Donde tal historia debe de contar con lugares específicos involucrados, al igual que en los tiempos de los hechos, y la importancia de las acciones de cada una de las personas intervinientes en los sucesos relevantes.

\section{UTILIDAD DE LA TEORIA DEL CASO.}

Tiene como principal utilidad la construcción de una investigación con un desarrollo eficaz, ya que se tiene conocimiento de un hecho delictuoso. La teoría del caso nos permitirá definir de la manera más coherente la versión que se tiene de los hechos sucedidos, ya que se tiene la obligación de realizar un análisis estratégico del caso y a su vez se tiene que hacer un ordenamiento de los hechos y así poder clasificar

\section{UNIVERSIDAD VERACRUZANA.}




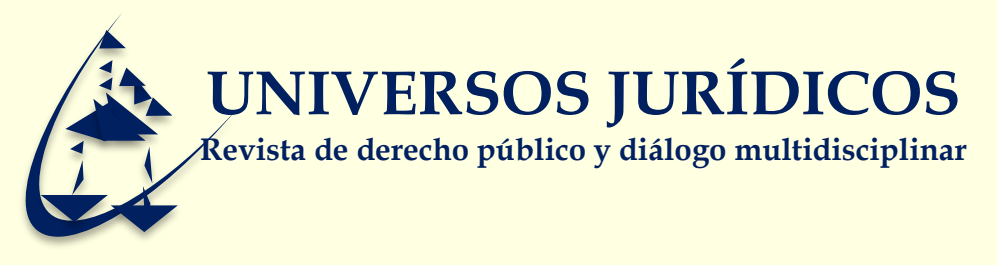

la información del caso Se tiene que determinar el objetivo fundamental de la investigación, en su caso seleccionar la evidencia más relevante con que se intenta comprobar este hecho delictuoso.

Con todo lo anterior se intenta evitar inconsistencias e incongruencias en la presentación de la teoría planteada, así mismo se busca el momento más oportuno para así poder presentar las pruebas necesarias para su caso. También se permitirá la dirección perfecta que se pueda dar al examen y contra examen, lo cual les dará a las partes el beneficio de saber que preguntas objetar y cuáles no.

Y es por eso que elaborar una teoría del caso, asegura una gestión profesional exitosa, por permitir controlar eficaz y coherentemente las diligencias y actividades procesales que le favorecen o perjudican a cada una de las partes.

Una buena teoría del caso debe poder explicar cómoda y consistentemente la mayor cantidad de hechos de la causa, incluidos aquellos que establezca la contraparte. La teoría del caso, en suma, es nuestra simple, lógica y persuasiva historia de "lo que realmente ocurrió". 


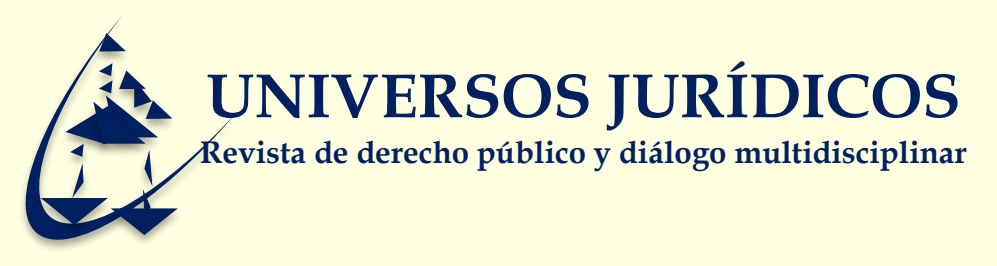

\section{SUJETOS PROCESALES}

\section{a. JUEZ.}

En su libro, el autor Arteaga Sandoval ,Miguel Ángel, establece que con motivo de la actual tendencia en materia procesal penal, la figura de juez penal sea transformado, rompiendo paradigmas, terminando con tradiciones y formalismos hoy considerados ociosos, tal es el caso del referendo secretarial; en el nuevo proceso penal desaparece orgánicamente de los órganos jurisdicciones la figura del secretario, como fedatario judicial, y de adjudica el juez de fe pública para el ejercicio de sus funciones, prescindiendo así de la cuenta y validación secretarial de la resolución judicial. (2013)

En este nuevo sistema de corte acusatorio y oral, se cuenta con una figura nueva que está sujeta a una suma de responsabilidades y es por eso que nace la necesidad de crear un nuevo perfil, donde éste debe de contar con criterios de profesionalismo, ética y tener una capacitación constante, con una abundante flexibilidad para el aprendizaje que la exige el nueva sistema de la impartición de justicia, al igual debe de estar apto para poder escuchar el derecho ante la doble 


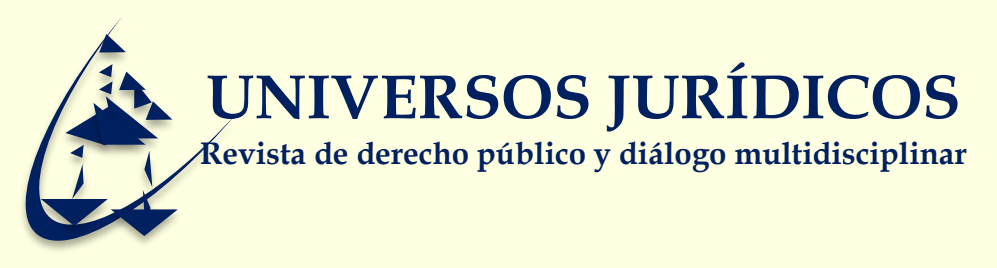

realidad del sistema acusatorio, a su vez tiene que contar con la habilidad suficiente para analizar toda la información que sea vertida en la audiencia, de igual forma tiene que adaptarse a un nuevo sistema de información tecnológica y lo más importante tiene que tener la suficiente capacidad de darle una dirección a la audiencia a través de un manejo adecuado cada uno de los elementos de los hechos controvertidos a través de la argumentación de cada una de las partes.

Se dice que este modelo de juez debe de contar con algunos elementos esenciales como los son, un sentido de improvisación y respuestas más rápidas en el coloquio judicial. Así como también contar con una manera específica y precisa en el análisis de la persona procesada, testigos, la defensa y al ministerio público para alcanzar a comprender la posición de cada uno de las partes, y así intentar resolver de la mejor manera posible dicha controversia, primero averiguando sobre los hechos y luego, subsumiendo esos hechos en una o más normas jurídicas que deberá aplicar, en esto, debe ser lo más firme posible con sus ideas y opiniones propias respecto de su sentido de justicia, sin dejar de lado la Constitución y la Ley.

Tiene la obligación de tomar la decisión del juicio, por lo que tiene que argumentar de manera imparcial y siendo honesto, es decir, con la capacidad de impartir 


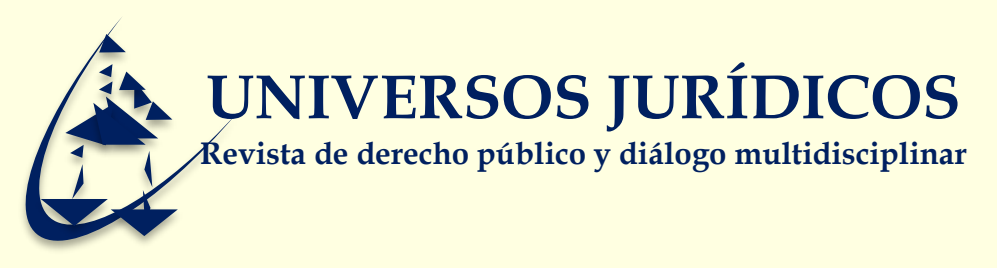

justicia, sin mirar nada más que a las propias y personales convicciones, las que nunca deberán ser arbitrarias, sino que, muy por lo contrarios elaborados, fundados y justificados en las leyes. Dicho razonamiento requiere una dosis de fuerza en la imparcialidad, porque por ninguna circunstancia debe de estar a favor de ninguna de las partes.

\section{b. DEFENSOR.}

Al lado del imputado se hace imprescindible la figura del defensor, el advocatus, el hombre de consejo, versado en la erudición del derecho, llamado al auxilio de aquel contra quien el estado pretende enderezar su acción persecutora, en ejercicio de su poder sancionador. La garantía de la defensa adquiere una relevancia particularmente trascendente, ya que, como expresión del debido proceso, ningún acto procesal en que intervenga el imputado será válido sin la asistencia de su defensor. (Arteaga, 2013)

El derecho a la defensa, es un derecho fundamental reconocido constitucionalmente y en los textos de derechos humanos, el cual debe salvaguardarse en cualquier procedimiento jurisdiccional. 


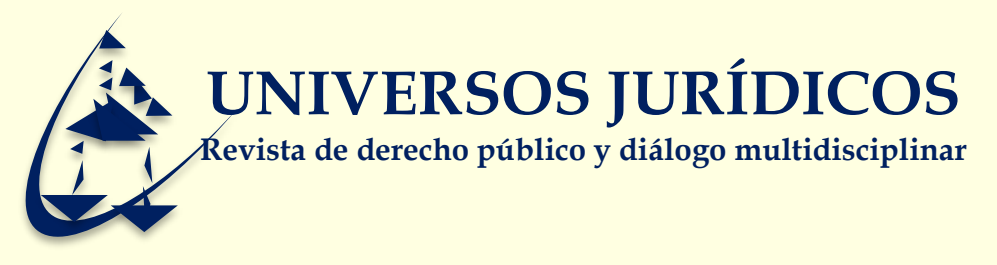

En la actualidad es un derecho fundamental e inviolable que tiene el imputado de poseer una defensa adecuada. Cuando no se cuente con los recursos económicos suficientes, en obligación del gobierno otorgar un defensor público, ya que en algunos casos no se tiene conocimiento de las leyes aplicables a tu caso, y se tendrá que contar con una defensa adecuada. Y este abogado tendrá la capacidad de resolver el caso con el objetico primordial de una resolución en beneficio del imputado. Así permitir responder al estado correctamente del proceso por el cual se le quiere castigar.

En el artículo 20 fracciones VIII y XI, apartado B, de la Constitución Política de los Estados Unidos Mexicanos, establece, Tendrá derecho a una defensa adecuada por abogado, al cual elegirá libremente incluso desde el momento de su detención. Si no quiere o no puede nombrar un abogado, después de haber sido requerido para hacerlo, el juez le designará un defensor público. También tendrá derecho a que su defensor comparezca en todos los actos del proceso y éste tendrá obligación de hacerlo cuantas veces se le requiera, y

XI; En ningún caso podrá prolongarse la prisión o detención, por falta de pago de honorarios de defensores o por cualquiera otra prestación de dinero, por causa de 


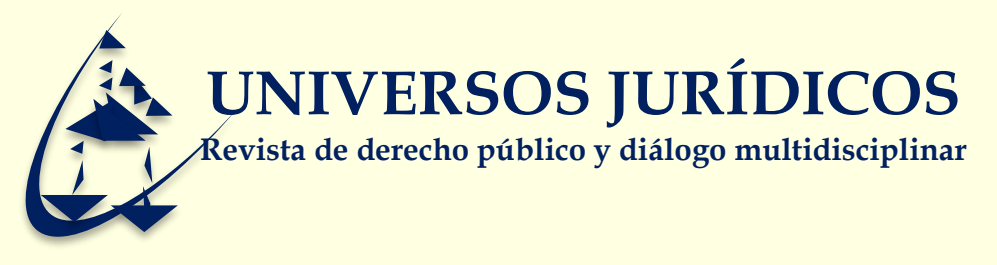

responsabilidad civil o algún otro motivo análogo. El defensor en materia penal se erige como el principal responsable del respeto a los derechos humanos y garantías contenidos en la Ley Suprema en favor del imputado. (Santa Cruz y Fernández, 2016).

El compromiso del abogado defensor es proteger la ley y sus principios en el resguardo de un cliente, y se hará una exigencia para que el abogado esté preparado para cuestionar las pruebas del Estado. Ninguna condena debe basarse en pruebas poco fidedignas, y por tanto el abogado defensor se ve obligado a investigar y cuestionar las pruebas y el pleito contra su defendido para asegurarse de que sea fiable. Los abogados defensores deben buscar los posibles u motivos por los que un testigo engaña e identificar y exponer las circunstancias que puedan comprometer la capacidad del testigo para observar, recordar o describir el evento de manera exacta.

La defensa adecuada se relaciona con el principio de contradicción, puesto que tiene que examinar los hechos y anteponer a las postulaciones y a los alegatos de la otra parte. Al igual que con el principio de presunción de inocencia, aunque éste 


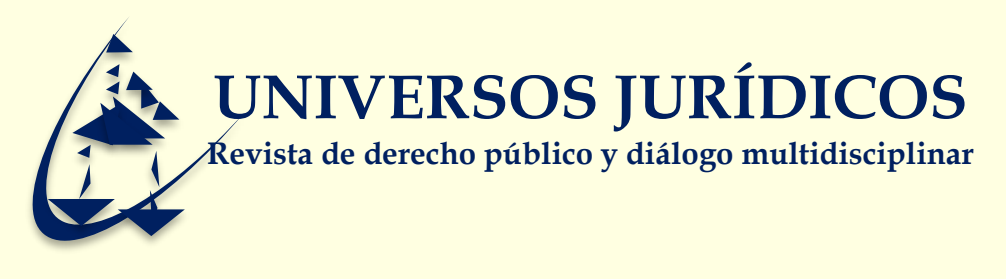

implica que la carga de la prueba es para el órgano acusador, la defensa deberá desvirtuar los datos que lo vinculen con un proceso penal.

\section{MINISTERIO PÚBLICO.}

El ministerio público es, un órgano estatal encargado de promover la justicia en defensa de la legalidad, de los derechos de los ciudadanos y del interés público tutelado por la ley, de oficios o instancia de los interesados, así como velar por la independencia de los tribunales y procurar ante esto la satisfacción de interés social.

Le corresponde al ministerio público representar los intereses de la sociedad afectada por la comisión de un hecho ilícito que le ley así lo señale. En el artículo 21 de la Constitución Política de los Estados Unidos Mexicanos a su letra dice, la investigación de los delitos corresponde al ministerio público y a las policías, los cuales actuaran bajo la conducción y mando de aquel en el ejercicio de esta función. 


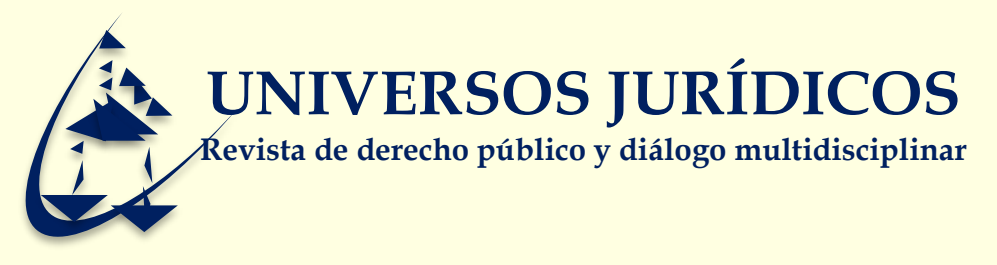

El ministerio público debe contar con conocimientos especializados en la procuración de justicia con la finalidad principal de crear una nueva gama de investigadores que dirijan la práctica de acciones y diligencias encaminadas a descubrir la verdad histórica. En este caso también se da una selección para el personal del ministerio público, esta tendrá que ser rigurosa, a su vez tendrán que someterse exámenes psicométricos. De conocimientos jurídicos, técnicos, y a su vez exámenes físicos.

Al igual en dicha selección se tomará en cuenta tanto como aptitudes como las actitudes de cada uno de los aspirantes. También se deberán instrumentar mecanismos que permitan la supervisión y evaluación de los constantes resultados.

\section{PONDERACIÓN}

La ponderación es una técnica para la interpretación de la Constitución y los derechos fundamentales, en definitiva es una práctica para la elucidación de principios, a veces pueden entrar en contradicción, por ejemplo la libertad con la igualdad y libertad de expresión, en estos casos la ponderación viene a medir o contrapesar cada uno de los principios, la cual se presenta como una habilidad que 


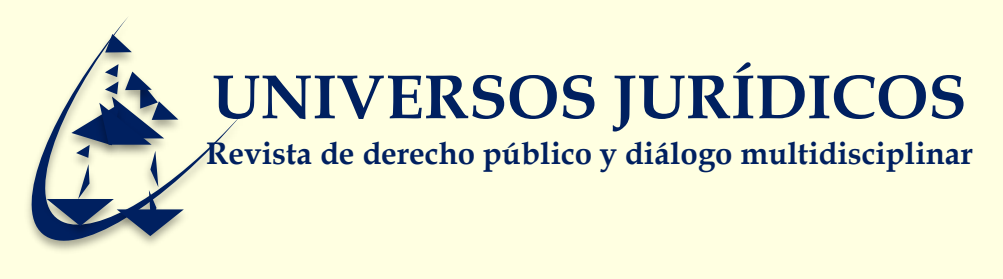

permite lograr la resolución de conflictos, lo que hace que los principios o reglas pueden valerse sobre otros, por lo que es un criterio de optimización de problemas

con la interpretación de los derechos. Es por eso que el juez está encargado de la ponderación en los juicios, al dar su veredicto tiene que seguir los principios y normas fundamentales de la Constitución en relación a la subjetividad y su razonamiento ante dicho delito.

Según Mocora Juan M." En líneas generales, se cree que la ponderación es una metodología adecuada para evaluar la corrección de los argumentos y decisiones que toman los jueces constitucionales en los casos de conflicto entre principios que expresan derechos fundamentales". (2017).

Los principios no son normas que establezcan exactamente lo que debe de hacerse, si no normas que exigen que algo sea realizado en la mayor medida posible, dentro de las posibilidades jurídicas y reales existentes. El ámbito jurídicamente posible está determinado por principios y reglas que juegan en 


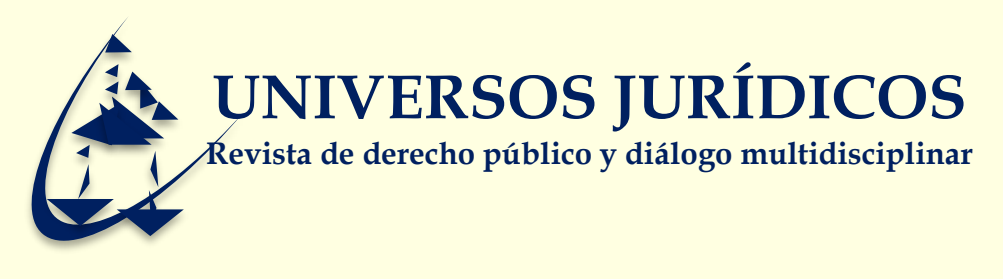

sentido contrario. Por su parte, los enunciados facticos acerca del caso determinan el ámbito de lo factible posible.

A fin de establecer la "mayor medida posible" en la que un principio debe de ser cumplido, es necesario contrastarlo con los principios que juegan en sentido contrario o con los principios que dan fundamentó a las reglas que juegan un sentido contrario. La ponderación representa el mecanismo para resolver esta incompatibilidad entre normas prima facie.

\section{CONCLUSIÓN.}

La teoría del caso se crea como una herramienta básica para que el hecho tenga el impacto necesario y con eso persuadir al juez, porque debe de existir un pensamiento lógico jurídico, ya que al momento de argumentar oralmente en las diferentes audiencias se pretende obtener el mayor beneficio, según sean las pretensiones procesales que se tengan.

Ya que, esta teoría se hace desde en el momento que se tenga conocimiento de los hechos, la cual tiene la finalidad de proporcionarle un significado u orientación 


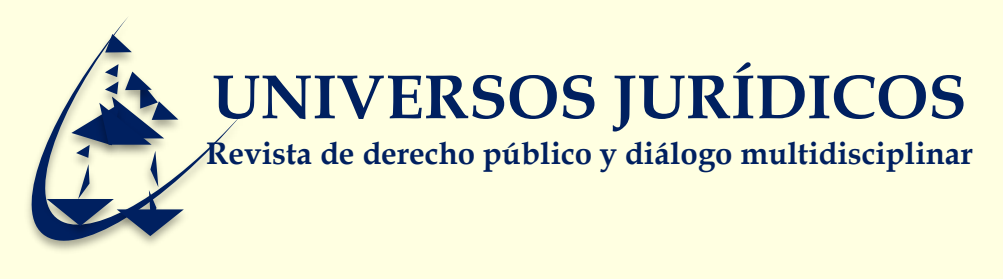

a los sucesos, así como a las normas jurídicas ya sean sustantivas o procesales, al igual que el material probatorio el cual se ha recabado.

Esta teoría es un instrumento metodológico que tienen las partes para así construir, recolectar, depurar y presentar su posición estratégica frente a los hechos materia del proceso; y por otro lado, es un procedimiento que permite la coyuntura de tres niveles de análisis: factico, jurídico y probatorio, ya que éstos no directamente benefician a las partes, sino al juez, ya que con esto, se le brindará una carga informativa que le permitirá contar con los elementos de juicio o decisión para solucionar el conflicto de interés jurídico.

Tal teoría también debe de contar con los planteamientos de las partes de los hechos sucedidos, se tiene que hacer con fundamentos legales y jurídicos. A su vez éstos se tienen que comprobar y hacer que el juez le dé una resolución a su favor, con cada elemento probatorio.

\section{REFERENCIAS}

Arteaga Sandoval, Ángel. (2013) Los sujetos procesales en el sistema penal acusatorio, México, editoriales flores.

Constitución Política de los Estados Unidos Mexicanos. 


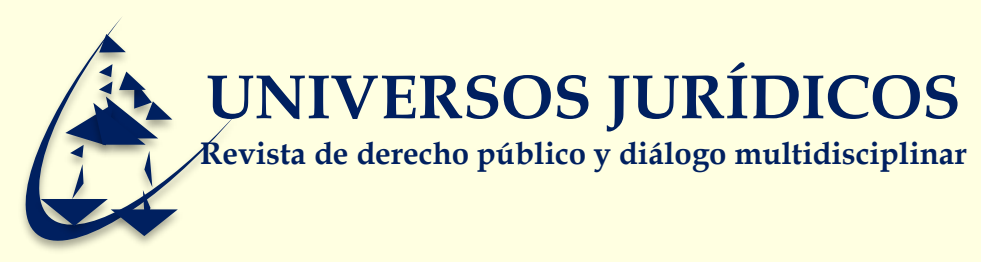

Hesbert Benavente, Chorres. (2012) La aplicación de la teoría del caso y la teoría del delito en el proceso penal acusatorio y oral, segunda edición, México, editorial Flores.

Martínez-Bastida, Eduardo, (2016) manual para litigantes del procedimiento nacional acusatorio y oral, editorial Raúl Juárez Carro, México.

Mocoroa, J.M. (2017). La racionalidad de la ponderación en la argumentación constitucional. Revista Prolegómenos Derechos y Valores, 20, 39, 73-85. DOI: http://dx.doi.org/10.18359/prole.2724

Ortiz Ruiz, José Alberto. (2014) Teoría del caso. Análisis y aplicación en los juicios orales en México, México, editorial Flores.

Santa Cruz Morales David y Fernández Santacruz Roberto. (2016) "Artículo de investigación "La importancia de la teoría del caso para lograr una defensa adecuada.

http://www.enciclopedia-juridica.biz14.com/.

http://www.juridicaformativa.uson.mx/memorias/v_coloquio/doc/derechoconstitucio nal/CAZAREZ_OLGA_Y_GERMAN_GUILLEN̄.pdf 